\title{
Exceptional Response to Olaparib in a Patient With Recurrent Ovarian Cancer and an Entire BRCA1 Germline Gene Deletion
}

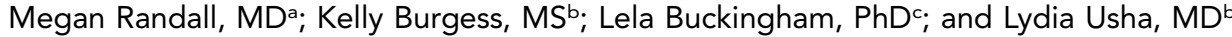

\section{ABSTRACT}

PARP inhibitors are known to be effective in patients with ovarian cancer (OC) and germline mutations in BRCA1 and BRCA2 genes (BRCA mutations). Little is known, however, about any correlation between the deletion size and location of the BRCA mutation and the response to PARP inhibitors. Women with OC commonly undergo genetic testing because the presence of a germline BRCA mutation impacts therapeutic decisions and is important for cancer surveillance in patients and their family members. This report presents a case of a rare entire germline BRCA1 gene deletion and an exceptional response to a PARP inhibitor, olaparib, in a heavily pretreated patient with OC. Her disease course was also remarkable for complete responses to platinum-based chemotherapy and long chemotherapyfree intervals. Interestingly, the deletion of the entire BRCA1 gene was found after previously negative BRCA test results and is associated with a deletion of 6 adjacent genes without known clinical significance. She has remained progression-free and asymptomatic for $>3$ years on olaparib, with an overall survival of $>12$ years. We postulate that this unusually favorable response and prolonged overall survival is related to the cancer cells' inability to reverse the entire gene deletion to wild-type (a common mechanism of resistance to PARP inhibition). This case shows the value of genetic testing for patients with $\mathrm{OC}$ and highlights the utility of additional testing with previously negative results and limited genetic testing. It also provides insight into a potential mechanism of an exceptional response to PARP inhibition.

J Natl Compr Canc Netw 2020;18(3):223-228 doi: 10.6004/jnccn.2019.7378

\footnotetext{
${ }^{a}$ Department of Internal Medicine; bivision of Hematology, Oncology, and Stem Cell Transplant Medicine, Department of Internal Medicine; and 'Department of Pathology, Rush University Medical Center, Chicago, Illinois.
}

PARP inhibitors such as olaparib, niraparib, rucaparib, and talazoparib comprise a new class of antineoplastic targeted agents that are FDA approved for the treatment of ovarian cancer (OC) and breast cancer. These agents are known to be most effective in patients with germline mutations in the BRCA1 and BRCA2 genes (BRCA mutations). ${ }^{1}$ Little is known, however, about any correlation between the size and location of the BRCA mutation and response to PARP inhibitors.

Genetic testing for germline BRCA mutations and other genomic alterations has become more common in recent years, with $>100,000$ Americans undergoing testing annually. ${ }^{2}$ Malignancies typically associated with $B R C A$ mutations include breast, ovarian, prostate, pancreatic, and melanoma. ${ }^{3}$ BRCA mutation status is important for the cancer surveillance of patients and their blood relatives, as well as for prognosis and treatment recommendations for carriers affected with cancer. Specifically, women with OC or breast cancer and germline $B R C A$ mutations are more likely to benefit from platinum-based chemotherapy and PARP inhibitors. ${ }^{1,4}$ All women diagnosed with OC should be referred for genetic counseling and testing, regardless of family history and age at diagnosis. ${ }^{5}$ The indication for genetic testing early in the course of the disease became stronger after FDA-approval of olaparib for use as maintenance after first-line platinum-based chemotherapy in women with $B R C A$-mutated OC. ${ }^{6,7}$

This report presents a case of a rare entire germline $B R C A 1$ gene deletion and an exceptional response to olaparib in a woman with advanced, heavily pretreated OC.

\section{Case Report}

A 43-year-old Hispanic woman without any known family history of malignancy (Figure 1) was diagnosed with stage III poorly differentiated ovarian adenocarcinoma in 2007. Initial treatment consisted of a total abdominal hysterectomy with bilateral salpingooophorectomy followed by adjuvant therapy with 6 cycles of carboplatin and docetaxel. She experienced a complete response to initial treatment. In 2010, she presented with abdominal pain and distention, and 


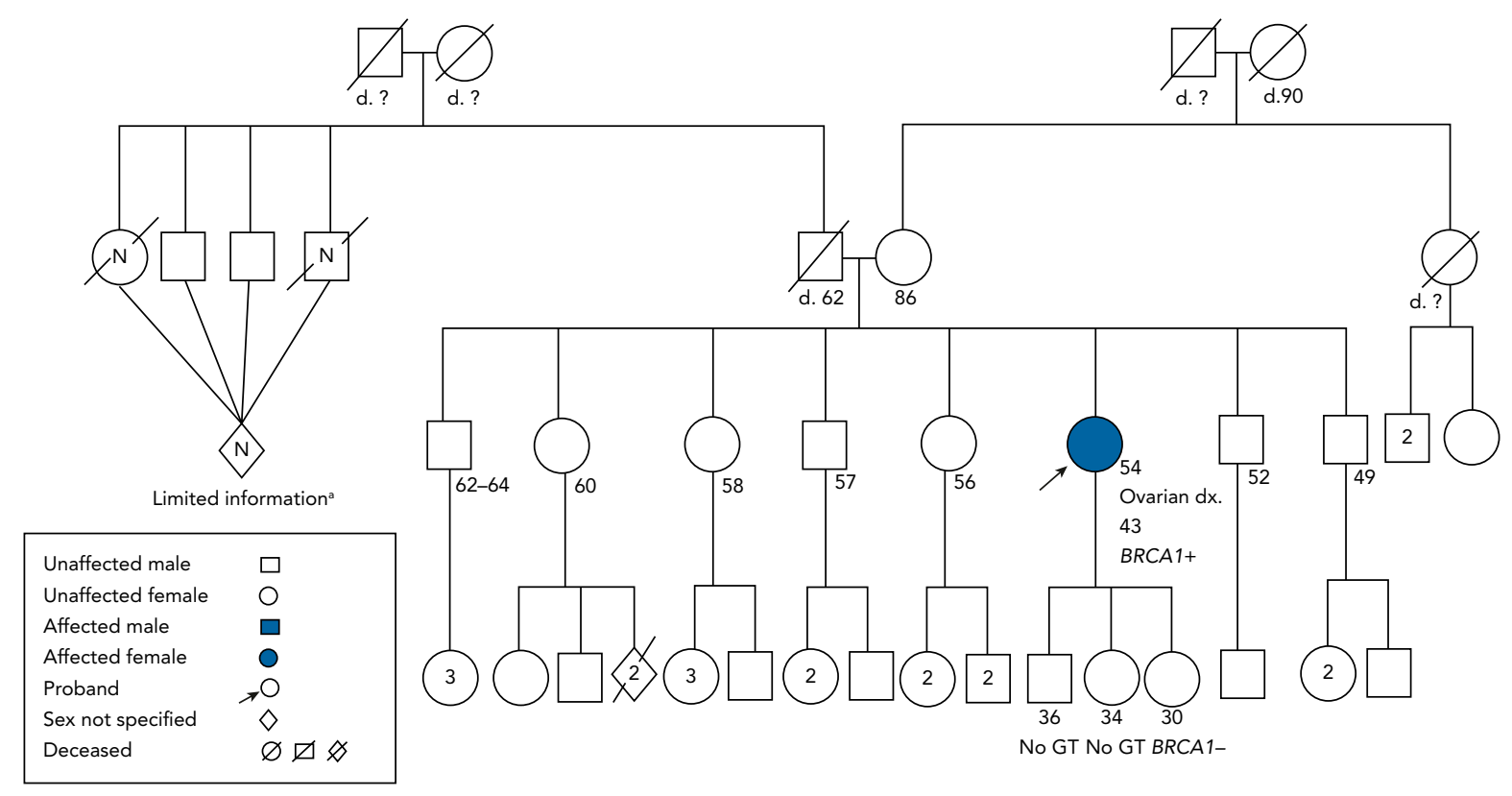

Figure 1. Patient pedigree. Both maternal and paternal ancestry were Mexican.

Abbreviations: d., death; d. ?, age of death unknown; dx, diagnosis; GT, genetic testing; N, number of individuals is unknown.

a Limited information is known regarding second-degree relatives and beyond. Because of this limitation, there could be additional cancer diagnoses in more extended relatives that are not known to the patient.

was found to have a recurrence. A CT scan revealed bilateral pleural effusions, ascites, and peritoneal metastases. She was treated again with 2 cycles of carboplatin and paclitaxel; however, she developed a hypersensitivity reaction to carboplatin with pruritus after the second cycle. She then underwent carboplatin desensitization and received 4 more cycles of carboplatin and liposomal doxorubicin (substituted for paclitaxel because of neuropathy) with complete response.

In December 2010, the patient underwent genetic testing through full-gene Sanger sequencing of the $B R C A$ genes and a 5-site rearrangement panel of the $B R C A 1$ gene (a targeted PCR panel used to detect 5 recurrent deletions and duplications of $\geq 1$ exons within the $B R C A 1$ gene). No germline $B R C A$ mutations were found.

She remained without evidence of disease until October 2012, when an increase in her CA 125 level was noted. A PET/CT scan revealed peritoneal implants and enlarged mesenteric lymph nodes. The patient again underwent 6 cycles of carboplatin on a desensitization protocol with liposomal doxorubicin and experienced a complete response. She was offered, but declined, bevacizumab maintenance.

In 2013, the patient underwent additional screening for full-gene rearrangement of the BRCA genes by multiplex ligation-dependent probe amplification, which analyzes multiple gene sequences in a single reaction and detects copy number variation, including deletions, of specific genes. This method revealed a deletion of the entire BRCAl gene.

She was monitored again with serial CA 125 measurements. Another recurrence was identified in 2015 through increased CA 125 levels. A PET/CT scan revealed progression with hypermetabolic supraclavicular, axillary, and retroperitoneal lymph nodes, as well as peritoneal metastases. She received 6 more cycles of carboplatin on a desensitization protocol with liposomal doxorubicin, which resulted in a complete response, and the patient was started on maintenance with olaparib in March 2016. She has continued on olaparib for $>3$ years and remains on it at the time of writing. She is clinically asymptomatic and working full-time. Her most recent CT scans of the chest, abdomen, and pelvis from November 2019 showed ongoing remission with no evidence of disease.

In 2019, the patient underwent additional genetic testing by chromosomal microarray, which uses comparative genomic hybridization and DNA microarray techniques to identify genomewide locus-by-locus deletions and duplications with high resolution. This test confirmed the presence of a complete $B R C A 1$ gene deletion and showed that the size of the deletion on chromosome $17 \mathrm{q}$ was larger than this one gene and measured $205 \mathrm{~kb}$. It included $B R C A 1$ and an additional 6 genes (Figure 2). Currently, none of the 6 surrounding 
genes involved in this deletion have known clinical significance independent of BRCA1.

The patient's daughter had a negative germline test result for the patient's BRCA1 gene deletion in 2018. No other family members have been tested.

\section{Discussion}

This patient's deletion of the entire BRCAl gene and a larger region of chromosome $17 \mathrm{q}$ is rare, with only a few cases reported in the literature. Her exceptional response to multiple lines of platinum-based combination chemotherapy and subsequent ongoing complete response to PARP inhibition with olaparib are also unusual. Although prognosis after an OC diagnosis may be poor for some patients, others have experienced longterm survival. ${ }^{8}$ Long-term survivors display favorable clinical features, including optimal cytoreductive surgery at diagnosis and platinum-sensitive disease indicated by a recurrence-free interval of $>12$ months after first-line chemotherapy ${ }^{9}$ In addition, patients with germline $B R C A$ mutations have clinical characteristics different from those of women with non-BRCA-related OCs, including younger age at presentation, high-grade histology, advanced stage at diagnosis, and better response to platinum-based chemotherapy. ${ }^{10}$ Large germline genomic rearrangements of $B R C A 1$ or BRCA2 regions at $17 \mathrm{q}$ or $13 \mathrm{q}$, respectively, are reportedly associated with more severe phenotype, including earlier onset of breast cancer (before 40 years of age), OC, bilateral breast cancer, and male breast cancer. ${ }^{11}$ Our patient was diagnosed with OC at the age of 43 years, which is younger than the average age of 51 years at OC diagnosis in BRCAl carriers, ${ }^{12}$ and could be the result of her rare mutation. The favorable effect of a $B R C A$ mutation on prognosis in patients with OC is fairly well established. Numerous publications, including a metaanalysis of 14 studies, have shown that patients with OC who have germline and somatic BRCA mutations have improved progression-free (PFS) and overall survival (OS). ${ }^{13,14}$

Our patient had an exceptional response to therapy in the context of an entire BRCAl germline gene deletion. Most BRCA mutations are point mutations (single nucleotide variants) or small deletions/insertions (frameshift) mutations. Large gene rearrangements (LGRs), including complete gene deletions or duplications, are relatively rare and constitute anywhere from $0 \%$ to $27 \%$ of $B R C A 1$ mutations. In most of the studied populations, LGRs represent $<10 \%$ of $B R C A 1$ mutations. ${ }^{11,15,16} \mathrm{~A}$ variation in prevalence of BRCA1 LGRs between different patient populations has been reported, with a significantly higher prevalence in patients from Latin America $(21.4 \%),{ }^{17,18}$ such as our patient, as well as in Dutch $(27 \%)$ and Italian $(20 \%)$ populations. ${ }^{16}$ These findings emphasize the importance of offering genetic testing, including LGR testing, for cancer predisposition to all women diagnosed with OC, regardless of their ethnic background.

Because of the rarity of LGRs, testing of the $B R C A$ genes was first performed through Sanger sequencing,

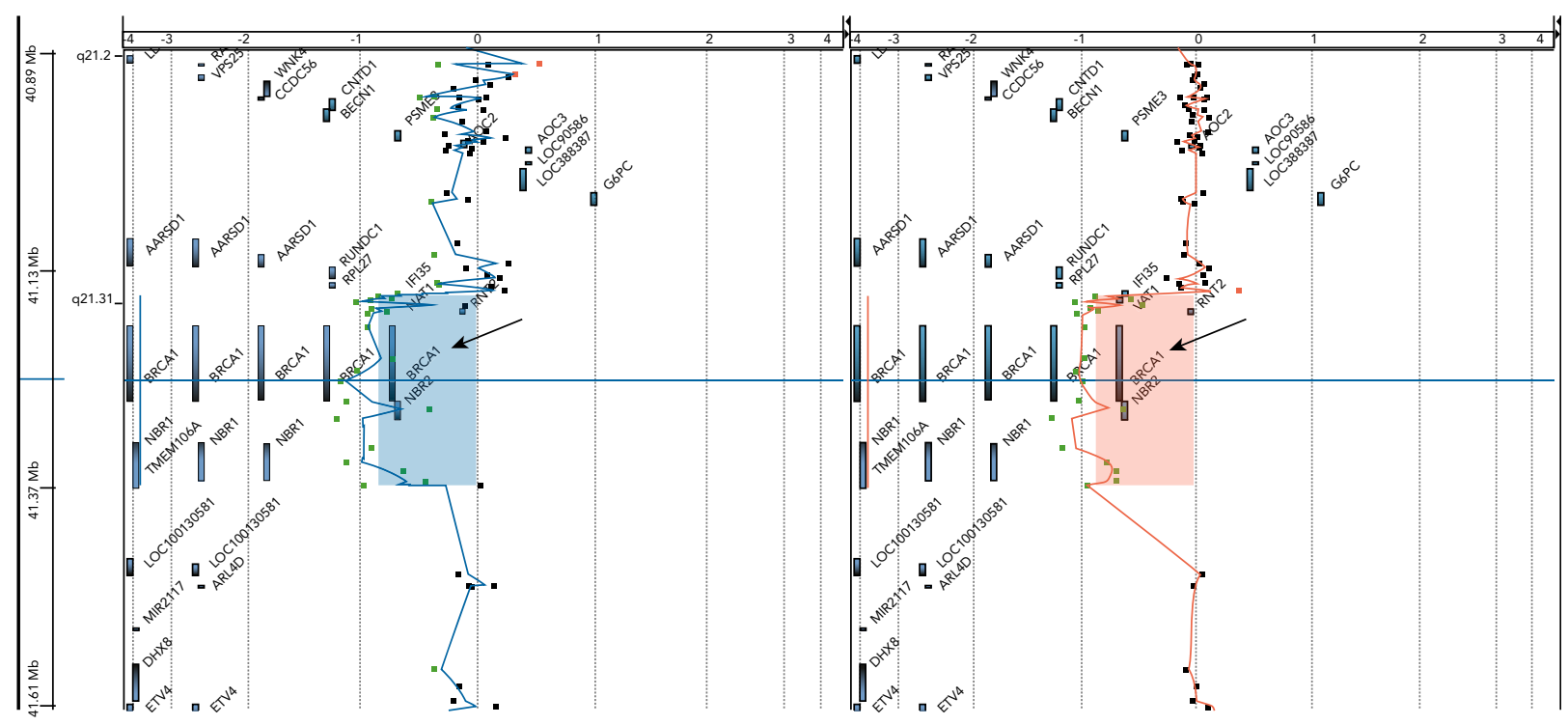

Figure 2. Results of the chromosomal microarray. Two runs were performed at the testing laboratory, and are represented by the blue and red diagrams. The blue and red boxes indicate the deletion present on chromosome 17. The arrows in each image point to the location of BRCA1. The diagram shows that the entire BRCA1 gene is deleted, and 6 other adjacent genes (IF135, VAT1, RND2, NBR2, NBR1, and TMEM106A) that are not currently thought to be clinically significant. 
the technology initially used for clinical identification of germline mutations in BRCA genes. It is a sequencingby-synthesis method and is a qualitative rather than quantitative analysis. ${ }^{19}$ This sequencing technique accurately detects point mutations but not LGRs. If a large gene sequence is present in one copy of the gene and not the other, as in a heterozygous germline deletion, Sanger sequencing will not detect any abnormality. Sanger sequencing does not detect gene dosage abnormalities, such as a complete gene deletion. In 2006, $B R C A$ testing using reverse transcription quantitative PCR (RT-qPCR) was implemented. RT-qPCR is a quantitative process and is able to detect LGRs by monitoring amplification of targeted DNA sequences. ${ }^{20}$ With these advances in technology and the introduction of next-generation sequencing, germline genetic testing methods can now detect point mutations, small deletions/insertions, and LGRs in one test.

Little is known about the effect of large or entire $B R C A$ gene deletions on treatment response, prognosis, or survival in patients with cancer. ${ }^{11,15,16}$ There are several case reports of patients with rare entire BRCAl germline deletions, although these reports do not describe the patients' clinical course or response to treatment. ${ }^{18,21-23}$ In one report, the entire BRCAl germline deletion in a Spanish woman with no family history was confirmed to be de novo. ${ }^{22}$ In the absence of cancer in her family, our patient's mutation may also represent a de novo mutation; however, her parents were not available for genetic testing to confirm this. Even more rare are entire
BRCA2 germline deletions. ${ }^{18,24,25}$ A single case report describes a patient with estrogen receptor-positive metastatic breast cancer and a large $B R C A 2$ gene deletion who had a complete response to olaparib. ${ }^{26}$

In patients with advanced OC and germline $B R C A$ mutations who have received at least 2 lines of previous chemotherapy, olaparib maintenance has been shown to prolong PFS compared with placebo (median, 19.1 vs 5.5 months; hazard ratio [HR], 0.30; 95\% CI, 0.22-0.41). ${ }^{27}$ Another study of maintenance olaparib in a similar population showed a trend toward improved OS in women on olaparib versus placebo (HR, $0.73 ; 95 \% \mathrm{CI}$, $0.45-1.17$ ) and a median OS of 34.9 months in women with germline $B R C A$ mutations. In this study, 15 patients (11\%) remained progression-free after 6 years on olaparib, although the mechanism of such a durable response remained unclear. ${ }^{28}$

Our patient's PFS of $>3$ years significantly exceeds the median PFS in these trials and represents an exceptional response to olaparib therapy. We believe that her improved outcome is related to the nature of her germline BRCA1 mutation. Several mechanisms have been proposed to explain the development of resistance to PARP inhibitors in BRCA mutation carriers. Reversion of the germline mutation to wild-type is one of the most important mechanisms (Figure 3). ${ }^{29,30}$ Although there may be other possible mechanisms to restore homologous recombination, we suggest that, as opposed to a point mutation or small insertion/deletion, an LGR/entire gene deletion is not easily amenable to

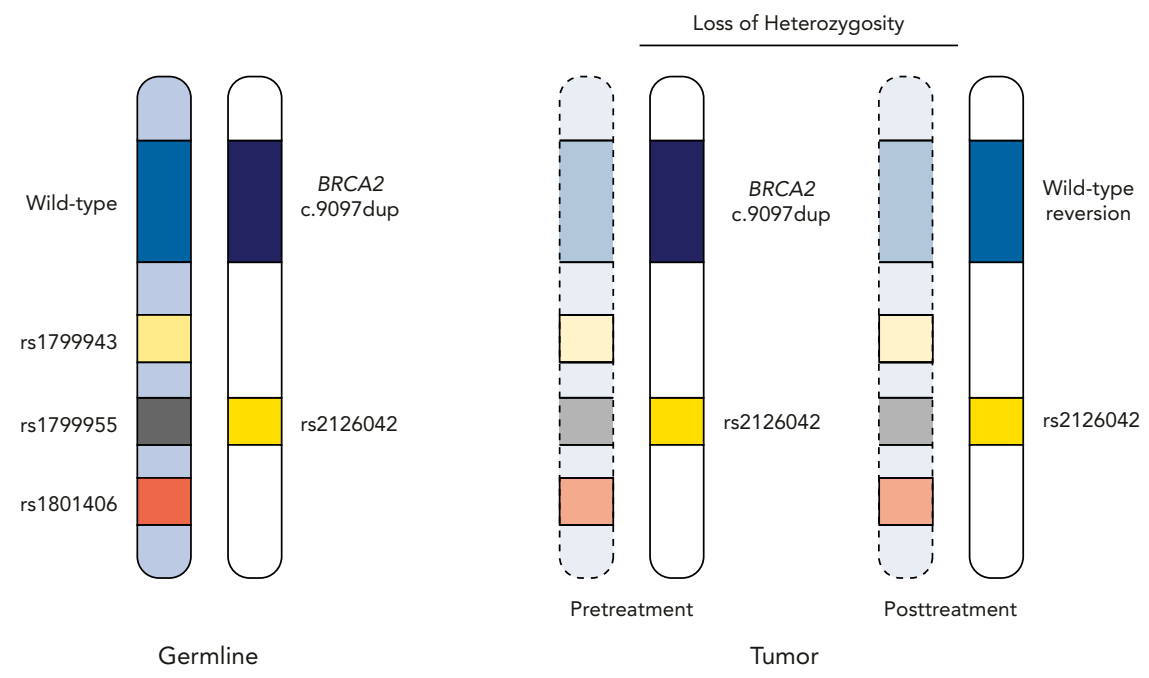

Figure 3. Mechanism of resistance to PARP inhibitors. Germline BRCA2 c.9097dup, with loss of the wild-type allele in the cancer, followed by a secondary reversion of the germline mutation to the wild-type in another allele after the patient's treatment with a PARP inhibitor, veliparib. In the lymphocytes (germline), the wild-type BRCE2 allele and the heterozygous reference single-nucleotide polymorphisms rs1799943, rs1799955, and rs1801406 were detected, along with the BRCA2 c.9097dup and a heterozygous single-nucleotide polymorphism, 2126042. Loss of heterozygosity of the wild-type BRCA2 was seen in the pretreatment sample, and the wild-type BRCA2 was restored by a reversion mutation in the posttreatment sample. From Banda K, Swisher EM, Wu D, et al. Somatic reversion of germline BRCA2 mutation confers resistance to poly(ADP-ribose) polymerase inhibitor therapy [published online February 14, 2018]. JCO Precis Oncol, doi: 10.1200/PO.17.00044. Reprinted with permission. @2020 American Society of Clinical Oncology. All rights reserved. 
reversion, and thus our patient's cancer cells retain sensitivity to olaparib. Moreover, her disease course was also characterized by an excellent response to 3 lines of platinum-based chemotherapy and long chemotherapy-free intervals. This exquisite sensitivity to platinum-based therapy may also be explained by the same inability of cancer cells to reverse the germline mutation to the wild-type BRCA1 gene. Because the patient remains disease-free, we are unable to repeat a tumor biopsy to confirm retention of the germline BRCA1 deletion.

As described earlier, our patient's germline deletion included 6 additional genes adjacent to the BRCAl gene; however, they are not presently known to affect the predisposition to cancer or response to therapy. Interestingly, among the deleted genes, $N B R 1$ (neighbor of $B R C A 1$ gene 1) encodes an autophagy receptor protein originally identified as an ovarian tumor antigen. ${ }^{31} \mathrm{An}-$ other gene in this region, NBR2 (neighbor of $B R C A 1$ gene 2) encodes a long noncoding RNA product and shares a bidirectional promoter with $B R C A 1 .{ }^{32}$ Amplification of 17p13.3-q25.3, including BRCA1, NBR1, and NBR2, has been observed and reported as pathogenic, suggesting that amplification rather than deletion of $N B R$ genes may affect phenotype. ${ }^{33}$ A 137.8-kb deletion, involving the first 6 exons of $B R C A 1$ and the full NBR2, BRCA1P1, NBR1, and TMEM106a genes, was detected in one case of highgrade serous OC; however, the pathogenic phenotype was attributed to loss of BRCAl function. ${ }^{34}$ Another deleted gene, $R N D 2$, is involved in regulating the migration and morphologic changes associated with the development of pyramidal neurons. ${ }^{35}$ This gene was included in a complete deletion of BRCAl associated with a case of breast cancer. ${ }^{22}$ Lastly, the VAT1 gene encodes for a synaptic vesicle integral membrane protein. ${ }^{36} \mathrm{~A}$ recent microarray analysis of differentially expressed genes between high-grade OC and normal ovarian epithelium identified VAT1 as one of several downregulated hub genes (common to multiple pathways) in OC. This study, however, did not report differential expression of BRCAl in these tumors. ${ }^{37}$

\section{Conclusions}

Our case of an unusually favorable response to olaparib in a heavily pretreated patient with $\mathrm{OC}$ with a rare germline abnormality-a deletion of the entire BRCAI gene-contributes to the understanding of PARP inhibitor mechanism of action. We think that this outstanding response and the long OS of $>12$ years is related to the inability of cancer cells to reverse such a large genomic rearrangement to the wild-type, which is a common mechanism of resistance to PARP inhibition as well as platinum compounds.

More studies are required to investigate an association between the size and specific location of $B R C A$ mutations and response to PARP inhibitors. However, given the rarity of such genetic abnormalities, it is unlikely that a study would be possible for women carrying germline LGRs or deletions of an entire BRCA gene. Thus, the experience described herein is important for genotype-phenotype correlations in the treatment of OC and can improve the care of these patients. Furthermore, our case underscores the value of genetic testing for all patients with OC, including the potential benefit of retesting in the setting of previously negative but limited genetic testing.

\section{Acknowledgments}

We acknowledge the generous contributions of Eugene and Shirley Deutsch, Mark and Maha Halabi Ditsch, George Ruwe, and Douglas and Sarah Criner, which made this work possible.

Submitted June 30, 2019; accepted for publication November 11, 2019.

Disclosures: The authors have disclosed that they have not received any financial consideration from any person or organization to support the preparation, analysis, results, or discussion of this article.

Correspondence: Megan Randall, MD, Department of Internal Medicine, Rush University Medical Center, 1700 West Van Buren Street, Suite 500, Chicago, IL 60612. Email: megan_e_randall@rush.edu

\section{References}

1. Faraoni I, Graziani G. Role of BRCA mutations in cancer treatment with poly(ADP-ribose) polymerase (PARP) inhibitors. Cancers (Basel) 2018; 10:487.

2. Armstrong J, Toscano M, Kotchko N, et al. Utilization and outcomes of BRCA genetic testing and counseling in a national commercially insured population: the ABOUT study. JAMA Oncol 2015;1: 1251-1260.

3. Mersch J, Jackson MA, Park M, et al. Cancers associated with BRCA1 and BRCA2 mutations other than breast and ovarian. Cancer 2015;121: 269-275.

4. Herzog TJ, Spetzler D, Xiao N, et al. Impact of molecular profiling on overall survival of patients with advanced ovarian cancer. Oncotarget 2016;7:19840-19849

5. Daly MB, Pilarski R, Berry MP, et al. NCCN Clinical Practice Guidelines in Oncology: Genetic/Familial High-Risk Assessment: Breast and Ovarian.

Version 3.2019. Accessed January 18, 2019. To view the most recent version, visit NCCN.org.

6. Moore K, Colombo N, Scambia G, et al. Maintenance olaparib in patients with newly diagnosed advanced ovarian cancer. N Engl J Med 2018;379. 2495-2505.

7. Kaufman B, Shapira-Frommer R, Schmutzler RK, et al. Olaparib monotherapy in patients with advanced cancer and a germline BRCA1/2 mutation. J Clin Oncol 2015;33:244-250.

8. Hoppenot C, Eckert MA, Tienda SM, et al. Who are the long-term survivors of high grade serous ovarian cancer? Gynecol Oncol 2018;148: 204-212.

9. Dao F, Schlappe BA, Tseng J, et al. Characteristics of 10-year survivors of high-grade serous ovarian carcinoma. Gynecol Oncol 2016;141:260-263.

10. Gadducci A, Guerrieri ME. PARP inhibitors alone and in combination with other biological agents in homologous recombination deficient epithelial 
ovarian cancer: from the basic research to the clinic. Crit Rev Oncol Hematol 2017;114:153-165.

11. James PA, Sawyer S, Boyle S, et al. Large genomic rearrangements in the familial breast and ovarian cancer gene BRCA1 are associated with an increased frequency of high risk features. Fam Cancer 2015;14 287-295.

12. Kotsopoulos J, Gronwald J, Karlan B, et al. Age-specific ovarian cancer risks among women with a BRCA1 or BRCA2 mutation. Gynecol Oncol 2018;150:85-91.

13. McLaughlin JR, Rosen B, Moody J, et al. Long-term ovarian cancer survival associated with mutation in BRCA1 or BRCA2. J Natl Cancer Inst 2013; 105:141-148.

14. Xu K, Yang S, Zhao Y. Prognostic significance of BRCA mutations in ovarian cancer: an updated systematic review with meta-analysis. Oncotarget 2017;8:285-302.

15. Lim YK, Lau PT, Ali AB, et al. Identification of novel BRCA large genomic rearrangements in Singapore Asian breast and ovarian patients with cancer. Clin Genet 2007:71:331-342.

16. Sluiter MD, van Rensburg EJ. Large genomic rearrangements of the BRCA1 and BRCA2 genes: review of the literature and report of a novel BRCA1 mutation. Breast Cancer Res Treat 2011;125:325-349.

17. Judkins $T$, Rosenthal $E$, Arnell $C$, et al. Clinical significance of large rearrangements in BRCA1 and BRCA2. Cancer 2012;118:5210-5216.

18. Fachal L, Blanco A, Santamariña M, et al. Large genomic rearrangements of BRCA1 and BRCA2 among patients referred for genetic analysis in Galicia (NW Spain): delimitation and mechanism of three novel BRCA1 rearrangements. PLoS One 2014;9:e93306.

19. Sanger F, Air GM, Barrell BG, et al. Nucleotide sequence of bacteriophage phi X174 DNA. Nature 1977;265:687-695.

20. Higuchi R, Dollinger G, Walsh PS, et al. Simultaneous amplification and detection of specific DNA sequences. Biotechnology (N Y) 1992;10: 413-417.

21. Konecny $M$, Zavodna $K$, Vranova $V$, et al. Identification of rare complete BRCA1 gene deletion using a combination of SNP haplotype analysis, MLPA and array-CGH techniques. Breast Cancer Res Treat 2008;109: 581-583.

22. Garcia-Casado Z, Romero I, Fernandez-Serra A, et al. A de novo complete BRCA1 gene deletion identified in a Spanish woman with early bilateral breast cancer. BMC Med Genet 2011;12:134.

23. de la Hoya M, Gutiérrez-Enríquez S, Velasco E, et al. Genomic rearrangements at the BRCA1 locus in Spanish families with breast/ovarian cancer. Clin Chem 2006;52:1480-1485.

24. Caux-Moncoutier V, Castéra L, Tirapo C, et al. EMMA, a cost- and timeeffective diagnostic method for simultaneous detection of point mutations and large-scale genomic rearrangements: application to BRCA1 and BRCA2 in 1,525 patients. Hum Mutat 2011;32:325-334.

25. Tournier I, Paillerets BB, Sobol $\mathrm{H}$, et al. Significant contribution of germline BRCA2 rearrangements in male breast cancer families. Cancer Res 2004; 64:8143-8147

26. Moiseyenko VM, Chubenko VA, Moiseyenko FV, et al. "Lazarus response" to olaparib in a virtually chemonaive breast cancer patient carrying gross BRCA2 gene deletion. Cureus 2018;10:e2150.

27. Pujade-Lauraine E, Ledermann JA, Selle F, et al. Olaparib tablets as maintenance therapy in patients with platinum-sensitive, relapsed ovarian cancer and a BRCA1/2 mutation (SOLO2/ENGOT-Ov21): a double-blind randomised, placebo-controlled, phase 3 trial. Lancet Oncol 2017;18: 1274-1284.

28. Friedlander M, Matulonis U, Gourley C, et al. Long-term efficacy, tolerability and overall survival in patients with platinum-sensitive, recurrent high-grade serous ovarian cancer treated with maintenance olaparib capsules following response to chemotherapy. Br J Cancer 2018;119:1075-1085.

29. Banda K, Swisher EM, Wu D, et al. Somatic reversion of germline BRCA2 mutation confers resistance to poly(ADP-ribose) polymerase inhibitor therapy [published online February 14, 2018]. JCO Precis Oncol doi: 10.1200/PO.17.00044

30. Ganesan S. Tumor suppressor tolerance: reversion mutations in BRCA1 and BRCA2 and resistance to PARP inhibitors and platinum [published online February 14, 2018]. JCO Precis Oncol. doi: 10.1200/PO.18.00001

31. Campbell IG, Nicolai HM, Foulkes WD, et al. A novel gene encoding a B-box protein within the BRCA1 region at 17q21.1. Hum Mol Genet 1994; 3:589-594.

32. Brown MA, Xu CF, Nicolai $\mathrm{H}$, et al. The $5^{\prime}$ end of the BRCA1 gene lies within a duplicated region of human chromosome $17 q 21$. Oncogene 1996;12:2507-2513.

33. National Center for Biotechnology Information. ClinVar; [VCV000602688.1] Available at: https://www.ncbi.nlm.nih.gov/clinvar/variation/NCV000602688.1. Accessed November 2019.

34. Rizza R, Hackmann K, Paris l, et al. Novel BRCA1 large genomic rearrangements in Italian breast/ovarian cancer patients. Mol Diagn Ther 2019:23:121-126.

35. Negishi M, Katoh $\mathrm{H}$. Rho family GTPases and dendrite plasticity. Neuroscientist 2005;11:187-191.

36. Mertsch S, Becker M, Lichota A, et al. Vesicle amine transport protein-1 (VAT-1) is upregulated in glioblastomas and promotes migration. Neuropathol Appl Neurobiol 2009;35:342-352.

37. Alur VC, Raju V, Vastrad B, et al. Mining featured biomarkers linked with epithelial ovarian cancer based on bioinformatics. Diagnostics (Basel) 2019;9:39. 